\title{
Actitudes lingüísticas en torno al pretérito en modo indicativo de estudiantes de la Universidad Nacional Mayor de San Marcos
}

\section{Linguistic attitudes towards the indicative mood past shown by students from Universidad Nacional Mayor de San Marcos}

\author{
Mirella Alexandra Robles Muñoz \\ Universidad Nacional Mayor de San \\ Marcos, Lima, Perú \\ mirella.robles@unmsm.edu.pe
}

\author{
Edgar Mauro Yalta Gonzales \\ Universidad Nacional Mayor de San \\ Marcos, Lima, Perú \\ edgar.yalta@unmsm.edu.pe
}

\begin{abstract}
Resumen
En el presente artículo, se describe cuáles son las actitudes lingüísticas que tienen los hablantes del español limeño - estudiantes de la Universidad Nacional Mayor de San Marcos (UNmSM) - respecto al pretérito en modo indicativo. Para tal propósito, se toma en cuenta la aplicación de los siguientes métodos: la encuesta, la entrevista sociolingüística y el Matched-Guise test, con la finalidad de recabar la frecuencia de uso del pretérito, conocer la competencia y las actitudes lingüísticas respecto al pretérito en modo indicativo por parte de los estudiantes de la UNMSM. Asimismo, se pretende comprobar las siguientes hipótesis: los estudiantes de la UNMSM no siempre emplean la lengua estándar al usar los pretéritos en contextos de narraciones y preguntas directas parciales. Además, los estudiantes de la UNMSM tienen actitudes e ideas positivas respecto al uso del pretérito en su variedad estándar y negativa en la no estándar. Por otro lado, la justificación radica en que los resultados brindarán una visión clara respecto a las actitudes lingüísticas de los hablantes de español limeño.
\end{abstract}

Palabras clave: Actitudes lingüísticas, Matched-Guise test, pretérito.

\begin{abstract}
This article describes the linguistic attitudes shown by Spanish speakers from Lima -students at the Universidad Nacional Mayor de San Marcos (UNMSM)- with respect to the past in indicative mood. For this purpose, the application of the following methods is taken into account: the survey, the sociolinguistic interview and the Matched-Guise test, in order to obtain the frequency of use of the preterite, to know the linguistic competence and attitudes with respect to the preterite in indicative mood by the students of the UNMSM. It is also intended to test the following hypotheses: UNMSM students do not always use the standard language when using preterites in contexts of partial narratives and direct questions. In addition, UNMSM students have positive attitudes and ideas regarding the use of preterite in its standard variety and negative in its non-standard variety. On the other hand, the justification lies in the fact that the results will provide a clear vision regarding the linguistic attitudes of the Spanish speakers from Lima.
\end{abstract}

Keywords: Linguistic attitudes, Matched-Guise test, preterite.

Recibido: 20.06.19

Aceptado: 15.07.19 


\section{Edgar Mauro Yalta Gonzales}

\section{Introducción}

En el presente artículo se pretende describir cuáles son las actitudes lingüísticas que tienen los estudiantes de la Universidad Nacional Mayor de San Marcos (UNMSM) respecto al pretérito en modo indicativo. El objeto de estudio es el pretérito en modo indicativo; para ello, se tomarán en cuenta tres aspectos importantes, que guardan relación con la frecuencia de uso, las competencias y actitudes lingüísticas que tienen los hablantes respecto a este tiempo verbal. Así, se pretende dar a conocer las valoraciones de los estudiantes, con respecto al uso de los tiempos verbales, tomando como referencia la lengua estándar. La justificación de este trabajo radica en que los resultados serán importantes para ofrecer una visión clara respecto a cuáles son las actitudes lingüísticas de los hablantes del español limeño, en este caso estudiantes de la Universidad Nacional Mayor de San Marcos (UNMSM), en torno al pretérito, todo esto en base a la observación de la variedad de uso y frecuencia de los tipos de pretérito en diversos contextos y la competencia de los estudiantes.

Una de las hipótesis que se plantea es que los estudiantes de la Universidad Nacional Mayor de San Marcos (UNMSM) no siempre emplean la variedad estándar al usar los pretéritos en contextos de narraciones y preguntas directas parciales. Muchos estudiantes de dicha casa de estudios consideran que la variedad estándar es la única forma correcta en que se debe desarrollar el uso del lenguaje. Por otro lado, la siguiente hipótesis es que los estudiantes de la UNMSM tienen actitudes positivas respecto al uso del pretérito en su variedad estándar y negativa en la no estándar.

En base a lo mencionado anteriormente, mediante la recopilación de los datos sobre el uso de los tiempos verbales se pretende recabar información que sustenten las hipótesis. Es por ello que empleando tres métodos se pretende realizar un análisis descriptivo (recopilar cantidades acerca de los usos de tiempos verbales y el conocimiento de la competencia de los hablantes); y otro, de carácter evaluativo (recopilar las actitudes de los estudiantes con respecto a los tiempos verbales). Esta hipótesis pretende contrarrestar las valoraciones de los estudiantes en torno al uso de los pretéritos que no siempre corresponden al empleo de la variedad estándar; y, sobre todo, reflexionar sobre ciertas estimaciones con respecto a que si existe o no el uso adecuado de la lengua.

Respecto a los estudios previos, En El perfecto simple y el perfecto compuesto en el español actual: estado de la cuestión de Silvia Hurtado Gonzales (1998), el objetivo de la autora es reunir ideas y cuestiones en torno al perfecto simple y el

88 Lengua \& Sociedad, vol. 18, n. ${ }^{\circ}$ 1, Lima, enero-junio de 2019 
Actitudes lingüisticas en torno al pretérito en modo indicativo de estudiantes...

perfecto compuesto del español actual. Para ello, toma en cuenta monografías, artículos y gramáticas donde se proporcionan información sobre el tema con el fin de explicar las características básicas de ambos tiempos verbales. Por otro lado, en El perfecto en el español de Lima. Variación y cambio en situación de contacto lingüístico de Margarita Jara (2013), la autora a lo largo de su publicación explora los valores del perfecto en narraciones de experiencias personales de hablantes del español limeño con la finalidad de estudiar la variación lingüística. Asimismo, los capítulos de la publicación brindan información muy relevante para la realización de este trabajo: el español de Lima y su caracterización, la sincronía y diacronía del pretérito perfecto y su uso en el español contemporáneo, el pretérito perfecto en la narrativa de experiencias personales, los factores lingüísticos y extralingüísticos en el uso del pretérito perfecto en la narraciones y, finalmente, el contacto lingüístico y formas verbales del pasado en el español hablado de Lima. Por último, en la tesis presentada por Clàudia Valls (2017) Usos del Pretérito Perfecto Simple y Compuesto en la Lengua Española: Un análisis comparativo de distintas variedades, la autora busca comprender el uso del pretérito perfecto simple y compuesto en distintas zonas de hispanohablantes tanto en su registro formal como informal. Para el desarrollo de la tesis, la autora utilizó encuestas a partir de usos y valores establecidos por la Real Academia Española, de esta manera pudo analizar y verificar la oposición de los usos del pretérito perfecto simple y compuesto entre el español de España y el español de América.

\section{Las actitudes lingüísticas y el pretérito}

Se pretende evaluar las actitudes de los estudiantes de la UNMSM en torno al pretérito en modo indicativo, tomando como referencia la lengua estándar. Para ello es importante considerar conceptos tales como actitud lingüística y MatchedGuise test. Comas (2006) manifiesta: «una actitud lingüística depende de lo que el hablante sabe acerca de las distintas funciones de la lengua según su propia experiencia y según las estrategias que utiliza para comunicarse con las personas de su comunidad o de otras comunidades» (p. 286). Entonces, para percibir dichas valoraciones por parte de los estudiantes, se realizó la técnica conocida como Matched-Guise test.

El Matched-Guise test, consiste en evaluar las distintas personalidades de las voces considerando pares ocultos (Hernández \& Sima, 2015). Asimismo, Hernández \& 


\section{Edgar Mauro Yalta Gonzales}

Sima (2015) añaden que la técnica ha recibido críticas y modificaciones notables. En primer lugar, los jueces no evalúan la lengua, si no el tema, la personalidad, la calidad de la grabación o el tipo de voz de los participantes. Entre las modificaciones, Hernández \& Sima (2015) mencionan que se pueden utilizar dos lenguas distintas o considerar el uso de distractores.

Según la RAE (2014), el pretérito es un tiempo que sitúa un estado, acción o proceso expresado por un verbo en un punto anterior al momento en que se habla. Asimismo, la RAE (2014) brinda una división del pretérito: pretérito anterior, pretérito imperfecto, pretérito perfecto compuesto, pretérito perfecto simple y pretérito pluscuamperfecto.

Respecto al pretérito anterior, la RAE (2014) lo define como el tiempo perfectivo que sitúa la acción expresado por un verbo en un punto pasado inmediatamente al anterior: hube cantado. El pretérito imperfecto es un tiempo imperfectivo que indica que la acción expresada por el verbo se está desarrollando de manera simultánea a cierta acción pasada: cantaba (RAE, 2014). La RAE (2014), define al pretérito perfecto compuesto como el tiempo perfectivo que sitúa la acción expresada por el verbo en un momento anterior al presente o en un lapso no concluido: he cantado. Por otro lado, el pretérito perfecto simple es un tiempo perfectivo en el cual la acción expresada por el verbo se sitúa a un momento anterior al momento del habla: cantaron (RAE, 2014). Finalmente, el pretérito pluscuamperfecto es un tiempo perfectivo que sitúa la acción expresada por el verbo en un momento anterior a otro igualmente pasado: había cantado (RAE, 2014).

\section{Metodología}

El estudio se realizó en hablantes de español limeño, específicamente en estudiantes de la Universidad Nacional Mayor de San Marcos de distintas facultades. Como ya se mencionó, se aplicó tres métodos distintos: la encuesta, la entrevista sociolingüística y el Matched-Guise test.

En primer lugar, la encuesta; se aplicó a veinte alumnos seleccionados de las carreras de la Facultad de Letras y Ciencias Humanas de la unMsm y de carreras de otras facultades tales como Contabilidad, Administración e Ingeniería. Además, se utilizó una ficha sociolingüística en cada estudiante encuestado con el propósito de realizar una comparación entre las variables de sexo, edad, carrera, ciclo, lugar de nacimiento e idiomas con respecto al uso de los pretéritos. La encuesta 
Actitudes lingüisticas en torno al pretérito en modo indicativo de estudiantes...

consistió en diez preguntas donde los estudiantes debían señalar como conjugarían el tiempo pasado tanto en contextos de preguntas directas parciales y de narraciones.

Por otro lado, en el caso de la entrevista sociolingüística, se pretendió conocer la competencia de los estudiantes de la UNMSM; es decir, si los alumnos encuestados aplican la norma al utilizar el pretérito en modo indicativo en base a las siguientes preguntas: ¿Qué hiciste en tu última navidad? y ¿Qué hiciste la semana pasada? En este método, se entrevistaron a nueve estudiantes de la UNMSM, los cuales fueron distintos a los encuestados del primer método empleado, incluyendo alumnos de otras facultades.

Finalmente, en el caso del método denominado Matched-Guise test, se pretendió conocer las actitudes lingüísticas de los alumnos de la UNMSM. En base a la presentación de dos textos (uno en variedad estándar y el otro no) que fueron leídos y evaluados por los entrevistados. Además, se presentaron adjetivos polares que fueron seleccionados por los entrevistados, es decir, evaluaron tanto al texto como a su redactor. Es importante mencionar que en este caso se aplicó el MatchedGuise test a los mismos entrevistados de la entrevista sociolingüística.

\section{Análisis}

En el presente apartado, se pasa a describir e interpretar los resultados obtenidos de cada uno de los métodos aplicados. Como ya se mencionó anteriormente, con cada método se buscó encontrar la frecuencia de uso, las competencias y las actitudes lingüísticas que tienen los hablantes respecto a este tiempo verbal.

\subsection{Encuesta: frecuencia de uso}

La presente encuesta tuvo como objetivo recabar información acerca de los usos lingüísticos en el habla natural de los estudiantes de la Universidad Nacional Mayor de San Marcos; es decir, la frecuencia de uso del pretérito en contextos de narración y preguntas directas parciales.

En las preguntas 2, 6 y 9 (narraciones), el tiempo que más se utilizó fue el imperfecto (26), seguido por el perfecto simple (16) y el pluscuamperfecto (16), es importante agregar que se ubicó un caso del pretérito anterior y pretérito perfecto compuesto. Asimismo, se encontró una constante en alumnos de cuarto ciclo de Lingüística por el uso del imperfecto en contextos de narración, además aquí se dio el único caso de uso del pretérito anterior el cual fue elegido en un 
contexto de pasado remoto («la semana pasada...»). El único caso del uso del perfecto compuesto desarrollado en un contexto, también, de pasado remoto. Por otro lado, otra constante fue que alumnos de sexto ciclo hasta décimo tanto de Lingüística como de otras carreras de la FLCH compartieron equitativamente el uso del imperfecto, el perfecto simple y el pluscuamperfecto.

Tabla 1. Frecuencia de uso de los tiempos verbales

\begin{tabular}{l|l}
\hline Tiempos verbales & Total \\
\hline Imperfecto & 33 \\
\hline Perfecto simple & 91 \\
\hline Perfecto compuesto & 12 \\
\hline Pluscuamperfecto & 61 \\
\hline Anterior & 3 \\
\hline Total & 200 \\
\hline
\end{tabular}

Fuente: Elaboración propia

Respecto al total de resultados, clara preferencia por el uso del perfecto simple y luego del pretérito pluscuamperfecto; sin embargo, el imperfectivo también marca distancia respecto a los otros tiempos restantes (pretérito anterior y perfecto compuesto). Es importante notar que, a pesar de ser el perfecto simple el más usado a nivel general, ello no corresponde con la mayor preferencia de uso que se tiene en contextos de narraciones porque es el pretérito imperfecto el más resaltante en este contexto. Si bien el uso del imperfecto es el que impera en contextos de narraciones, ello no ocurre en contextos de preguntas directas con alternativas, ya que es el segundo (después del pretérito anterior) en menor uso de preferencia con respecto a los tiempos verbales. Mientras que, en los contextos de narración el perfecto simple y el pluscuamperfecto presentan igual cantidad, en los contextos de preguntas directas hay una marcada diferencia entre estos dos tiempos verbales con una mayor preferencia en el uso del perfecto simple. Finalmente, es importante señalar que el uso del pretérito anterior fue exiguo en comparación con los otros tiempos al solo aparecer tres veces, una en narración $\mathrm{y}$ dos en preguntas.

92 Lengua \& Sociedad, vol. 18, n. ${ }^{\circ}$ 1, Lima, enero-junio de 2019 
Actitudes lingüísticas en torno al pretérito en modo indicativo de estudiantes...

\subsection{Entrevista sociolingüística: competencia de los estudiantes}

La entrevista sociolingüística tuvo como objetivo dar a conocer la competencia lingüística de los estudiantes de la Universidad Nacional Mayor de San Marcos; es decir, si los encuestados aplican la variedad estándar al utilizar el pretérito en modo indicativo en base a las preguntas: ¿Qué hiciste tu última Navidad? y ¿Qué hiciste la semana pasada?

En base a las gramáticas consultadas, los usos correctos de los tiempos en pretérito dependen de lo que el emisor quiere expresar. Por ejemplo, para el uso del perfecto simple se debe expresar un hecho concluido; para el imperfecto, en hechos habituales; en el caso del pluscuamperfecto, hechos anteriores a otros concluidos; el compuesto, hechos cuyas consecuencias se mantienen y el anterior, hechos inmediatamente anteriores a otro concluido.

En el caso de los estudiantes de Lingüística, el emisor cumple con el uso correcto de los pretéritos, especialmente en el uso del perfecto simple como el imperfecto al momento de narrar una situación concluida y una que se acción que se mantiene. En ambos casos, utilizan correctamente los tiempos según la normativa. Por otro lado, en el caso de los estudiantes de otras carreras de la Facultad de Letras y Ciencias Humanas, utilizaron los mismos tiempos: perfecto simple e imperfecto, además es relevante señalar que lo utilizaron de manera correcta. Es importante mencionar también que se encontraron los primeros casos de un perfecto compuesto. Finalmente, en el caso de los encuestados de otras facultades de la Universidad Nacional Mayor de San Marcos, alumnos relacionados a las carreras de negocios, utilizan de manera correcta el perfecto simple. En el caso de encuestado relacionado a la carrera de medicina, utilizó tiempos compuestos y utilizó de manera correcta el perfecto simple e imperfectivo. Finalmente, el estudiante relacionado con la carrera de ingeniería utilizó de manera correcta el perfecto simple limitándose, en todo momento de la entrevista, solo al uso de ese tiempo.

\subsection{Matched-Guise test: actitudes lingüísticas}

La aplicación del Matched-Guise test tuvo como objetivo dar a conocer las actitudes lingüísticas de los estudiantes de la Universidad Nacional Mayor de San Marcos. Es decir, si los encuestados tienen actitudes positivas o negativas respecto al pretérito en modo indicativo en base a dos textos, uno en variedad estándar y el otro en variedad no estándar. Se realizó a los mismos estudiantes de la entrevista 


\section{Edgar Mauro Yalta Gonzales}

sociolingüística y se utilizó adjetivos polares (culto-inculto, bien escrito-mal escrito, bueno-malo, urbano-rural, instruido-no instruido, adulto-joven, etc.) con la finalidad de determinar las ya mencionadas actitudes.

Las actitudes que muestran los estudiantes, en torno a los dos textos presentados (el primero escrito en lengua estándar y, el segundo escrito en lengua no estándar), son muy diversas y refieren a dos sentidos, principalmente. Todas las evaluaciones califican tanto al redactor de ambos textos, como al texto en sí, de tal forma que se establezca alguna relación entre los conocimientos que posee el escritor (con respecto al correcto o incorrecto uso de los pretéritos) con lo escrito.

En el texto 1 (narración escrita en variedad estándar), la mayoría de estudiantes califica, tanto al redactor como a la narración en sí, de forma positiva. Sin embargo, también se perciben algunas contradicciones en las respuestas; por ejemplo, cuando uno de los entrevistados (estudiante de Ingeniería) considera que el redactor es instruido, pero el texto está mal escrito. Otro caso se presenta cuando dos de los entrevistados (estudiantes de Arte y Literatura) coinciden al considerar que el texto es bueno, pero está mal escrito. La misma valoración presenta el entrevistado estudiante de Comunicación, solo que de forma opuesta, malo y bien escrito.

Los entrevistados de las carreras de Lingüística y Contabilidad consideran que un correcto uso de los pretéritos puede ser redactado, incluso, por una persona joven y, que un adulto puede redactar un mal texto (encuestado estudiante de Administración). El encuestado estudiante de Radiología, considera que no necesariamente el redactor debe poseer un alto cargo para escribir un buen texto. Por último, los estudiantes encuestados de Ingeniería y Lingüística consideran que el correcto uso de los pretéritos solo puede ser escritos por personas con alta experiencia (adulto), alto conocimiento (culto e instruido) y de acomodada clase social (rico, urbano e importante).

En el texto 2 (narración escrita en variedad no estándar), los estudiantes presentan calificativos tanto positivos como negativos con respecto al redactor y a la narración. Las contradicciones más resaltantes que se perciben al calificar a la narración y al redactor, son proferidas por los estudiantes encuestados de Contabilidad, Ingeniería y Radiología. Los primeros estudiantes (Contabilidad e Ingeniería) consideran al redactor como culto e instruido, pero que redacta un texto mal escrito; por otro lado, el estudiante de Radiología considera al redactor como inculto, pero que a la vez redacta un buen texto. Los estudiantes de Arte y Literatura consideran que un texto bien escrito no depende de la condición social del redactor; es decir, personas que viven en zonas rurales pueden realizar

94 Lengua \& Sociedad, vol. 18, n. ${ }^{\circ}$ 1, Lima, enero-junio de 2019 
Actitudes lingüisticas en torno al pretérito en modo indicativo de estudiantes...

un correcto uso de los pretéritos. Los estudiantes encuestados de Lingüística y Administración fueron tajantes en sus evaluaciones, el primero considera que el uso incorrecto de los pretéritos se manifiesta por personas de escasa capacidad intelectual y baja condición social; mientras que, el estudiante de Administración considera que un correcto uso de los pretéritos se realiza por personas cultas y de alta categoría social.

Es importante considerar que no todos los estudiantes tienen noción acerca de la variedad estándar; sin embargo, con conocimiento o sin ello, sus respuestas permiten evaluar las actitudes que presentan frente al uso de los pretéritos, si consideran que una persona con alta capacidad intelectual y, con alta posición social debe, necesariamente, escribir un buen texto o no. $\mathrm{O}$, si consideran que, por ejemplo, una persona de escasos recursos, puede poseer un gran conocimiento (a pesar de no poder acudir a una escuela) y; por lo tanto, hacer un buen uso de los pretéritos. Todas esas evaluaciones fueron presentadas de manera sucinta en el análisis.

\subsection{Limitaciones}

Algunas dificultades se suscitaron, especialmente, en la elaboración, durante y después de la aplicación de los métodos (parte de análisis). En la formulación de la encuesta (método 1), por ejemplo, las respuestas a las preguntas presentadas no arrojaron resultados que respondan con los objetivos previstos en el trabajo; sino, únicamente, nos permitió realizar un análisis descriptivo sobre nuestro objeto de estudio (los pretéritos). Ello nos llevó a elaborar un segundo método, la entrevista sociolingüística, la cual se realizó en base a la formulación de dos preguntas que tan solo nos permitió evaluar las competencias que poseen los estudiantes entrevistados, más no un análisis completamente evaluativo con respecto a los pretéritos. Por último, se realizó un tercer método (Matched-Guise test) con el objetivo de acceder a las actitudes que poseen los estudiantes respecto al uso correcto o incorrecto de los pretéritos. Sin embargo, también ocurrieron dificultades, por ejemplo, muchos estudiantes no evaluaban directamente el objeto de estudio, sino se dejaban llevar por otros aspectos, como el contenido de los textos presentados. Sin embargo, se pudo relacionar los resultados de los tres métodos utilizados. 


\section{Edgar Mauro Yalta Gonzales}

\section{Conclusiones}

Respecto a la frecuencia de uso del pretérito, en el total de los resultados, se observó una clara preferencia por el uso del perfecto simple seguido del pluscuamperfecto, así ambos marcan una distancia considerable sobre los otros tipos de pretérito. Por otro lado, luego de los tipos ya mencionados, sigue el imperfectivo que también traza una diferencia notoria respecto al perfecto compuesto y el pretérito anterior (el de menor frecuencia).

Los estudiantes se rigen al uso del estándar al momento de responder las preguntas; es decir, al narrar situaciones tanto de un pasado más lejano y uno cercano. Además, mencionar el frecuente uso de los tiempos perfecto simple e imperfectivo de manera correcta para los contextos de narración. Esto, contrarresta la hipótesis planteada, en la cual los estudiantes de la Universidad Nacional Mayor de San Marcos no siempre utilizan de forma correcta estos tiempos verbales.

Las actitudes lingüísticas que fueron evaluadas en base al método del MatchedGuise test permiten recabar información de los estudiantes, con respecto a cómo califican a las personas que hacen uso, de manera correcta e incorrecta, de los pretéritos. Así manifiestan (una minoría de los encuestados), tanto para el texto en variedad estándar como para el texto en variedad no estándar, que aquellos que usan correcta o incorrectamente los pretéritos, no dependerá de su alta o baja condición social, respectivamente. Sin embargo, algunos estudiantes consideran que sí existe tal correspondencia, de tal forma que estigmatizan las personas en base al uso correcto e incorrecto de los pretéritos.

\section{Referencias bibliográficas}

Castillo, M. (2006). El estudio de las actitudes lingüísticas en el contexto sociocultural: El caso del mexicano de Cuetzalan. Anales de Antropología, 40, 283-317. Hurtado, S. (1998). El perfecto simple y el perfecto compuesto en el español actual: Estado de la cuestión. EPOS, 16, 51-67.

Jara, M. (2013). El perfecto en el español de Lima (Variación y cambio en situación de contacto lingüístico). Lima: Pontificia Universidad Católica del Perú.

Real Academia Española. (2014). Diccionario de la lengua española. Recuperado de https://dle.rae.es.

Sima, E. \& Hernández, E. (2016). Una aplicación de Matched-Guise para el estudio de actitudes en lenguas en contacto de la península de Yucatán: maya, español e inglés en contextos urbanos. Estudios de Cultura Maya, 47, 151-180.

96 Lengua \& Sociedad, vol. 18, n. ${ }^{\circ}$ 1, Lima, enero-junio de 2019 
Actitudes lingüisticas en torno al pretérito en modo indicativo de estudiantes...

Valls, C. (2017). Usos del Pretérito Perfecto Simple y Compuesto en la Lengua Española: Un análisis comparativo de distintas variedades (Tesis de Grado en Filología Hispánica). Universidad de Barcelona, Barcelona. 